\title{
Modelo de interoperabilidad de la historia clínica electrónica utilizando HL7-CDA basado en computación en la nube
}

\author{
Víctor R. Velázquez M., Mabel Vázquez Briseño, Juan Iván Nieto H., \\ Juan de Dios Sánchez L. \\ Universidad Autónoma de Baja California, Facultad de Ingeniería Arquitectura y Diseño, \\ Baja California, México \\ \{vvmejia, mabel.vazquez, jnieto, jddios\}@uabc.edu.mx
}

\begin{abstract}
Resumen. La implementación exitosa de la Historia Clínica Electrónica (HCE) ayuda a mejorar la calidad de la atención de los pacientes, actualmente existe el problema de interoperabilidad entre las instituciones de salud, debido al uso variado de metodologías y tecnologías para su desarrollo. La Arquitectura de Documento Clínico (CDA) desarrollado por HL7 es un estándar de documentos para garantizar la interoperabilidad, la propagación de este formato de documento es fundamental para la interoperabilidad. El modelo presentado propone el desarrollo de sistemas orientados a la gestión de conocimiento clínico basándose en la generación de documentos CDA y su integración a través de la computación en la nube. Los desarrolladores que manejan diferentes plataformas podrán utilizar este modelo para mejorar la interoperabilidad. Los resultados obtenidos fueron la implementación de un modelo de uso general para desarrollo de HCE usando sintaxis basadas en XML para representar el conocimiento clínico mediante plantillas CDA.
\end{abstract}

Palabras clave: Interoperabilidad, historia clínica electrónica, HL7, CDA, computación en la nube.

\section{Introducción}

Actualmente en México las instituciones de salud utilizan sistemas de información clínica que no son compatibles entre sí, la información que se almacena en estos sistemas debería ser única y estar disponible en todo lugar al momento de prestar la atención médica a un paciente. Es transcendental que exista la forma de compartir la información clínica de un paciente para otorgarle una atención médica con calidad, es por ello que es importante buscar procesos y mecanismos técnicos que permitan desarrollar HCE con las características deseables mencionadas anteriormente, las cuales garanticen el cumplimiento de estándares clínicos, la capacidad de ser accesibles en cualquier momento y desde cualquier lugar, aun con el uso de diferentes tecnología para su desarrollo.

La Historia Clínica Electrónica (HCE) es "La recolección longitudinal de información electrónica de salud de una persona, donde la información de salud se 
define como la información relativa a la salud de un individuo o de atención sanitaria prestada a un individuo y puede apoyar los procesos eficientes para la prestación de asistencia sanitaria" [1]. Si se quiere garantizar el uso eficiente de la HCE, es indispensable que existan sistemas que logren el intercambio de información clínica, actualmente la mayoría de los sistemas son incompatibles. La interoperabilidad de las HCE se podría lograr si los sistemas de salud se desarrollan apegados a estándares de salud [2].

La interoperabilidad desde el punto de vista de la salud médica es "La habilidad de los sistemas para trabajar juntos, en general gracias a la adopción de estándares. La interoperabilidad no es solamente la habilidad de intercambiar información sanitaria, sino que requiere la habilidad de entender lo que se está intercambiando" [3].

Mejorando la interoperabilidad se tendrán mayores beneficios, tanto para los pacientes, como para los profesionales de la salud, ya que los registros médicos del paciente serían unificados. Con el uso de la Arquitectura de Documento Clínico como núcleo de los sistemas de salud se acercaría más a la interoperabilidad, esto se puede ver a través de diversos trabajos basados en CDA que de acuerdo con se han desarrollado con éxito en diferentes países [3].

La Arquitectura de Documento Clínico (CDA) de Health Level 7 (HL7), "es un estándar basado en XML para el marcaje de documentos que especifica la estructura y semántica de documentos clínicos para el propósito de facilitar su intercambio en un entorno de interoperabilidad" [4]. La estructura de la CDA es compleja y producir un documento correcto es difícil si no se conoce a fondo su estructura, además, las plataformas de las HCE entre las instituciones de salud varían tanto que cada institución de salud requeriría de su propio sistema generador de CDA. Las institución tienen su propias bases de datos para almacena su información, una de las características de la interoperabilidad es compartir la información entre instituciones, por esta razón, es importante almacenar los documentos utilizando sistemas que sean accesibles por las diferentes instituciones, una de ellas puede ser la utilizada por la computación en la nube.

La computación en la nube "es un sistema informático basado en Internet y centros de datos remotos para gestionar servicios de información y aplicaciones". La computación en nube permite a los consumidores y las empresas gestionar archivos y utilizar aplicaciones sin necesidad de instalarlas en cualquier computadora, requiriendo únicamente acceso a Internet. Esta tecnología ofrece un uso mucho más eficiente de recursos, como almacenamiento, memoria, procesamiento y ancho de banda, al proveer solamente los recursos necesarios en cada momento [5].

Actualmente existe un gran interés por promover la HCE utilizando CDA. El Servicio Nacional de Salud del Reino Unido inició un sistema de la HCE a nivel nacional, el proyecto hace uso del estándar HL7/CDA y SNOMED-CT [6]. En Canadá, la Canadian Health Infoway apoya el proyecto para la creación de un framework llamado Electronic Health Record System [7], el cual provee una estructura para el registro de salud electrónico, consiste de un conjunto de servicios comunes y reutilizables basados en SOA y HL7, lo que le permite tener estandarizadas las interfaces, las bases de datos, el vocabulario, entre otros elementos. En Latinoamérica también se han realizado trabajos en el área de interoperabilidad, tal es el caso del Libro Blanco de Interoperabilidad de gobierno electrónico para América latina V3.0 [8], en 
la cual se presenta la conceptualización de una arquitectura y una plataforma de interoperabilidad para América Latina y el caribe.

Con lo anteriormente expuesto, se puede visualizar que en algunos países ya se cuenta con plataformas de salud que utilizan estándares, permitiendo así la interoperabilidad entre las instituciones de salud y sus diferentes actores, algunos otros países tienen proyectos en desarrollo con grandes avances. Sin embargo, en México, a pesar de algunos desarrollos tecnológicos concretos, no existe un avance significativo en la definición formal de un modelo para la interoperabilidad de la HCE [9].

En este trabajo se presenta un modelo para generar e integrar documentos CDA en diferentes plataformas de desarrollo, los cuales pueden ser almacenados en diferentes hospitales además de la nube. El modelo incluye una herramienta que hace uso de documentos clínicos basados en el estándar HL7/CDA para el intercambio electrónico de información clínica y CDA para la sintaxis de las plantillas de los documentos clínicos los cuales están basado en XML. Este trabajo también ha tomado en cuenta la norma NOM-024-SSA3-2010 propuestas por la Secretaría de Salud [10]. Se propone por una parte la definición de aspectos para la implementación de la HCE, es decir, los expertos del dominio clínico definen el modelos de la información que será ingresada al sistema en forma de plantilla CDA y que posteriormente serán transformados a formatos web para ser llenados por los profesionales de la salud. Por otra parte, se propone una herramienta con los elementos mínimos requeridos para la administración de catálogos y módulos propuesta por la Secretaría de Salud, para esto se ha tomado en cuenta la Guía para el Desarrollo de Documentos CDA [11], la Guía de implementación HL7 para los datos de identificación de los pacientes [12].

Los beneficios de adoptar este modelo es que es un modelo de uso libre y los desarrolladores podrán utilizar la plataforma de desarrollo preferida. Por otra parte, Los hospitales que ya cuente con HCE podrán hacer uso del mismo con pocas modificaciones a sus sistemas, dado que es un modelo de uso libre, los hospitales podrán adoptarla.

El resto del documento está estructurado de la siguiente manera: tras esta introducción, se abordan los materiales y la metodología utilizados. A continuación se explica el modelo de interoperabilidad propuesto, posteriormente de presentan los resultados, para finalmente realizar una discusión y establecer las conclusiones.

\section{Materiales y métodos}

En esta sección, se presenta el modelo propuesto, se explican los componentes y técnicas necesarias para su implementación, se expone la generación e integración de las plantillas CDA basadas en la computación en la nube.

Se eligió el modelo de información CDA de HL7 Versión 2 para la representación de los documentos clínico debido a que es un modelo estándar, además de su gran simplicidad para su implementación en software, también fue considerada su sencillez tanto en su estructura de datos en memoria como en su estructura de base de datos. Es importante mencionar que la elección del modelo de la información clínica puede realizarse con base a las necesidades de la HCE que se esté implementando. Un documento CDA está dividido en dos partes, la Cabecera (HEAD) y el cuerpo (BODY). La cabecera tiene una estructura claramente definida, incluye información del paciente, 
hospital, médico, etc. El cuerpo es más flexible, contiene el reporte clínico, y está conceptualmente dividido en secciones anidadas, cada una de ellas contiene un bloque narrativo para ser presentado junto a entradas estructuradas y referencias externas [13]. Dentro de los documentos CDA existen categorías en función de su finalidad, para el modelo se utilizó el documento de atención CCD, ya que contiene el resumen de la información de la salud del paciente además de ser ampliamente utilizado para la interoperabilidad. Para el documento CDA se usó la información propuesta por la Dirección General de Información de Salud para la elaboración de la HCE.

La utilización de estos estándares por otras instituciones es importante, ya que es precisamente es lo que le da soporte y sustento a las herramientas de la propuesta, ya que las herramientas aquí presentadas apoyan a la creación de nuevas plantillas basadas en los actos clínicos que los expertos en el dominio clínico requieren para sus consultas médicas.

\subsection{Modelo de interoperabilidad propuesto}

La Fig. 1 muestra la arquitectura general del modelo de interoperabilidad para las HCE. El modelo está fundamentado en la gestión del conocimiento clínico, formado por roles con tareas bien definidas, artefactos en forma de insumo y la de generar y compartir documentos CDA compartidos entre hospitales, el intercambio de los documentos CDA se realiza a través de computación en la nube.

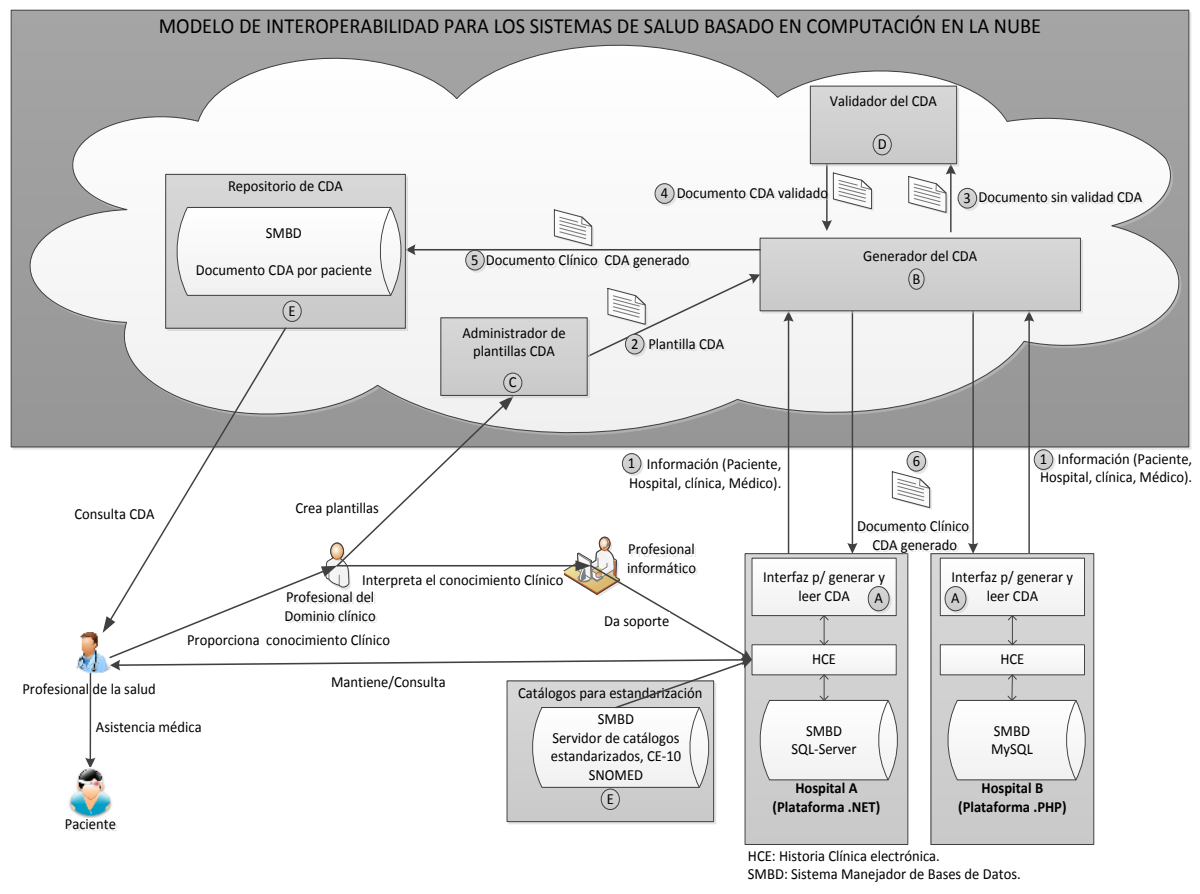

Fig. 1. Arquitectura para el modelo de interoperabilidad de la HCE. 
Los roles involucrados en el modelo son tres: El primero es el profesional de la salud quien tiene una participación activa en este modelo, este profesional es el experto en el conocimiento médico y es el usuario final del sistema. El segundo es el profesional del dominio clínico, quien debe poseer tanto conocimiento médico como conocimiento de las herramientas y normas que serán aplicadas a la HCE, este rol sirve como medio de comunicación entre los otros dos roles con el fin de evitar malas interpretaciones del conocimiento médico y las normas de salud, una de sus tareas es generar las plantillas CDA requeridas. Finalmente, el profesional informático quien no tiene experiencia en el conocimiento médico, pero si en las herramientas de modelado del conocimiento y en las herramientas para el desarrollo de software, su tarea es transformar a modelos informáticos lo que el profesional del dominio le transmite y dar soporte a los sistemas de las HCE.

En la Fig. 1 se muestra como los hospitales A y B cuentan con HCE desarrolladas en diferentes plataformas (.NET y PHP) y almacenan la información en distintos SMBD (Sql-Server y MySQL). Por lo cual se muestra que al utilizar este modelo es factible generar y compartir documentos CDA independientemente de la plataforma en que se haya desarrollado los sistemas que administran las HCE. Para lograr la interoperabilidad, en cada hospital será necesario contar con la interfaz para generar y leer documentos CDA, el proceso general para generar y leer documentos CDA es el siguiente:

A. Interfaz CDA: Sirve para para generar los documentos CDA enviando los datos del Paciente, el profesional de salud, el hospital, etc. al generador del documento CDA que se encuentra en la nube, además de recuperar los datos elaborados por generador documentos CDA.

B. Generador CDA: Utiliza los datos recibidos para crear el documento CDA acorde al formato de la plantilla deseada, la plantilla CCD es recuperada del administrador de plantillas y se encarga de llenar los campos correspondientes a la plantilla CCD con los datos que se encuentra organizado en dos secciones principales, la cabecera y el cuerpo del documento CDA de acuerdo a [13].

C. Administrador de plantilla: Contiene las plantillas necesarias de la HCE, estas plantillas son creadas por el profesional del dominio clínico según los requerimientos de una HCE, para esto se usan documentos CCD [13].

D. Validador CDA: Recibe el documento CDA generado, lo analiza para determinar si cumple con las normas especificadas por el esquema del CDA, una vez validado se regresa al generador de CDA, que a su vez lo envía al hospital receptor a través de la interfaz CDA. Adicionalmente, el documento es enviado al repositorio de documentos CDA para su organización y almacenamiento. Si no se valida, se devuelve un error y no se almacena el documento.

E. Repositorio CDA: Recibe el documento CDA validado, lo almacena y ordena de manera cronológica para cada paciente. Estos documentos podrán ser recuperados por los profesionales de la salud para consulta.

F. Servidor de catálogos: Proporciona información estandarizada para crear las HCE, esta información se refiere a los contenidos en SNOMED [14], LOINC [15], bases de datos geográficos, etc. 


\section{Resultados}

En esta sección, se presentan los resultados correspondientes a la implementación del modelo para la interoperabilidad de la HCE utilizando documentos clínicos HL7-CDA basado en computación en la nube.

\subsection{Entorno para generar plantillas CDA en la nube}

Para la plataforma de la nube se utilizó un servidor instalado en la Facultad de Ingeniería Arquitectura y Diseño de la Universidad Autónoma de Baja California, el cual cuenta con el sistema operativo Windows 8.0 de Microsoft, como servidor de base de datos se utilizó MS-SQLServer 2010, para la generación, integración y distribución de los documentos CDA se utilizó la plataforma de desarrollo de Microsoft Visual Studio 2010, específicamente el leguaje C\# y el framework de .NET 4.0, la plataforma de servicios web utilizado fue la de Internet Information Server (IIS) de Windows 8.0.

\subsection{Generación de plantillas CDA}

En el modelo no se determina que información será ingresada al sistema por los profesionales de la salud, sino que los profesionales del dominio clínico definen que conceptos clínicos desean manejar y que información será necesaria para cada tipo de acto clínico.

Para la prueba del concepto se crearon las plantillas CDA para el acto clínico de primer visita médica, para ello se tomó como información de entrada los datos del paciente establecidos en la norma oficial mexicana NOM-024-SSA3-2010, correspondiente al expediente clínico electrónico, esta información se analizó y se cruzó con la información clínica del estándar HL7-CDA V2, el profesional del dominio clínico creó y grabó estas plantillas en el administrador de plantillas CDA.

\subsection{Generación e integración de documentos CDA para la $\mathrm{HCE}$ del paciente}

Se crearon dos sistemas para la atención médica básica del paciente. En el primer caso se utilizó una plataforma compuesta por MS Visual Studio 2010 con lenguaje C\#, manejador de bases de datos SqlServer2010 y el servidor web IIS. En el segundo caso se utilizó WampServer 2.4, con el lenguaje de programación PHP 5.4.12, MySql 5.6.12 como administrado de bases de datos y Apache 2.4.4 como servidor web.

En ambos sistemas, los profesionales informáticos crearon interfaces para generar los formularios web basadas en las plantillas CDA almacenadas en la nube, las cuales serán llenadas por los profesionales de la salud en un acto médico determinado. Posteriormente, los documentos clínicos se depositan en un repositorio documental para que puedan ser recuperados por algún profesional de la salud con el fin de dar atención médica a un paciente, o bien, para ser compartidos con otra instancia médica.

Con el fin de garantizar la integridad de los documentos CDA utilizó la herramienta de validación de documentos CDA de Estados Unidos (NIST- http://cdavalidation.nist.gov/cda-validation), así mismo, con el fin de dar trazabilidad en la autoría de los documentos CDA se utilizó XML, ya que este permite la inclusión de la firma electrónica del profesional de salud que generó el documento. 


\section{Discusión y conclusiones}

El modelo propuesto ayuda a mejorar la interoperabilidad, los formatos de los documentos CDA son diseñados por los profesionales del dominio clínico, utilizando estándares clínicos HL7/CDA cruzados con los elementos mínimos recomendables por la Secretaría de Salud a través de la norma oficial mexicana NOM-024-SSA3-2010. Lo cual garantiza la interoperabilidad entre los sistemas de salud que adopten este modelo.

La propuesta tiene varias ventajas sobre otros proyectos existentes. Una de ellas es que las instituciones de salud que adopten el modelo no necesariamente tienen que adquirir un software propietario para generar e integrar documentos CDA almacenados en la nube, por lo que no tiene costos adicionales. La propuesta es de código abierto y puede ser utilizado independientemente de la plataforma de desarrollo que se utilice, ya que las plantillas están basadas en XML. El hecho de compartir información en la nube puede implicar un mejor control en la creación de plantillas CDA, estos es, si existe una plantilla que cumple con un acto clínico determinado, no hay necesidad de crear una nueva. Cuando se crean nuevas plantillas, no será necesario cambios en el software del lado del cliente; solo será necesario hacer las actualizaciones del lado del servidor, esto se realiza en la nube.

Como trabajo futuro se tienen que realizar pruebas exhaustivamente en la seguridad del servicio y su uso con accesos masivos de la herramienta en la nube. Las pruebas de seguridad realizadas están basadas en un módulo de accesos seguro al sistema, el cual está limitado a usuarios que tengan una cuenta con su clave de acceso y sus respectivas credenciales de uso, además de la seguridad en las bases de datos que ofrecen las propias herramientas en que fueron desarrollados.

\section{Referencias}

1. Carnicero, J., Fernández, A.: Manual de salud electrónica para directivos de servicios y sistemas de Salud. Santiago de Chile, Naciones Unidas (2012)

2. DGIS, Secretaría de Salud: Manual del Expediente Clínico Electrónico, México, (2011)

3. Institute of medicine, Patient Safety: Archiving a New Standard for Care. National Academy Press, Disponible en http://www.nap.edu/openbook.php?isbn=0309090776.

4. Subcomité técnico ADT-HL7 España: Guía para el desarrollo de documentos CDA. (2007)

5. Armbrust, M., Fox, A., Griffith, R., Joseph, A.D., Katz, R., Konwinski, A., Lee, G., Patterson, D., Rabkin, A., Stoica, I., Zaharia, M.: A view of cloud computing. Communications of the ACM, Vol. 53, No. 4, pp.50-58 (2010)

6. Department of Health Informatics Directorate UK. NHS Connecting for Health (NHS CFH). Consultado en Noviembre 2013 en http://www.connectingforhealth.nhs.uk. (2004)

7. Canada Government. Canada Health Infoway, https://www.infoway-inforoute.ca/index.php/ resources (2001)

8. Naciones Unidad-CEPAL: Libro blanco de interoperabilidad de gobierno electrónico para América Latina y el Caribe, Ver. 3.0 (2007)

9. Política Digital: Primer avance hacia el expediente clínico electrónico nacional, http://www.politicadigital.com.mx/?P=leernoticia\&Article=1175\&c=34. (2015)

10. Diario Oficial de la Federación: Norma Oficial Mexicana NOM-004-SSA3-2010, México http://www.dof.gob.mx/nota_detalle.php?codigo=5272787\&fecha=15/10/2012. (2015) 
Víctor Velázquez, Mabel Vázquez, Juan Nieto, Juan Sánchez

11. Subcomité Técnico ADT-HL7: Guía para el desarrollo de documentos CDA V1.0. España (2007)

12. Subcomité Técnico ADT-HL7: Guía de implementación HL/ Datos de Identificación del Paciente V.3 (2007)

13. HL7: CDA Release 2 http://www.hl7.org/implement/standards/product_brief.cfm? product_id=7 (2014)

14. International Health Terminology Standards development organization: SNOMED-CT User Guide July 2013 International Release (US English). http://ihtsdo.org/fileadmin/ user_upload/doc/download/doc_UserGuide_Current-en-US_INT_20130731.pdf. (2015)

15. McDonald, C., Stan, H, Jamalynne, D.: Logical Observation Identifiers Names and Codes Users' Guide LOINC. Recuperado en enero de 2014 de https://loinc.org/downloads/ files/LOINCManual.pdf. (2014) 